Document downloaded from:

http://hdl.handle.net/10251/120704

This paper must be cited as:

Hervás-Peralta, J.; Barrera Vilar, D.; Madrigal-Madrigal, J.; Sales Maicas, S. (2018).

Microwave Photonics Filtering Interrogation Technique Under Coherent Regime For Hot

Spot Detection on a Weak FBGs Array. Journal of Lightwave Technology. 36(4):1039-1045. https://doi.org/10.1109/JLT.2018.2793161

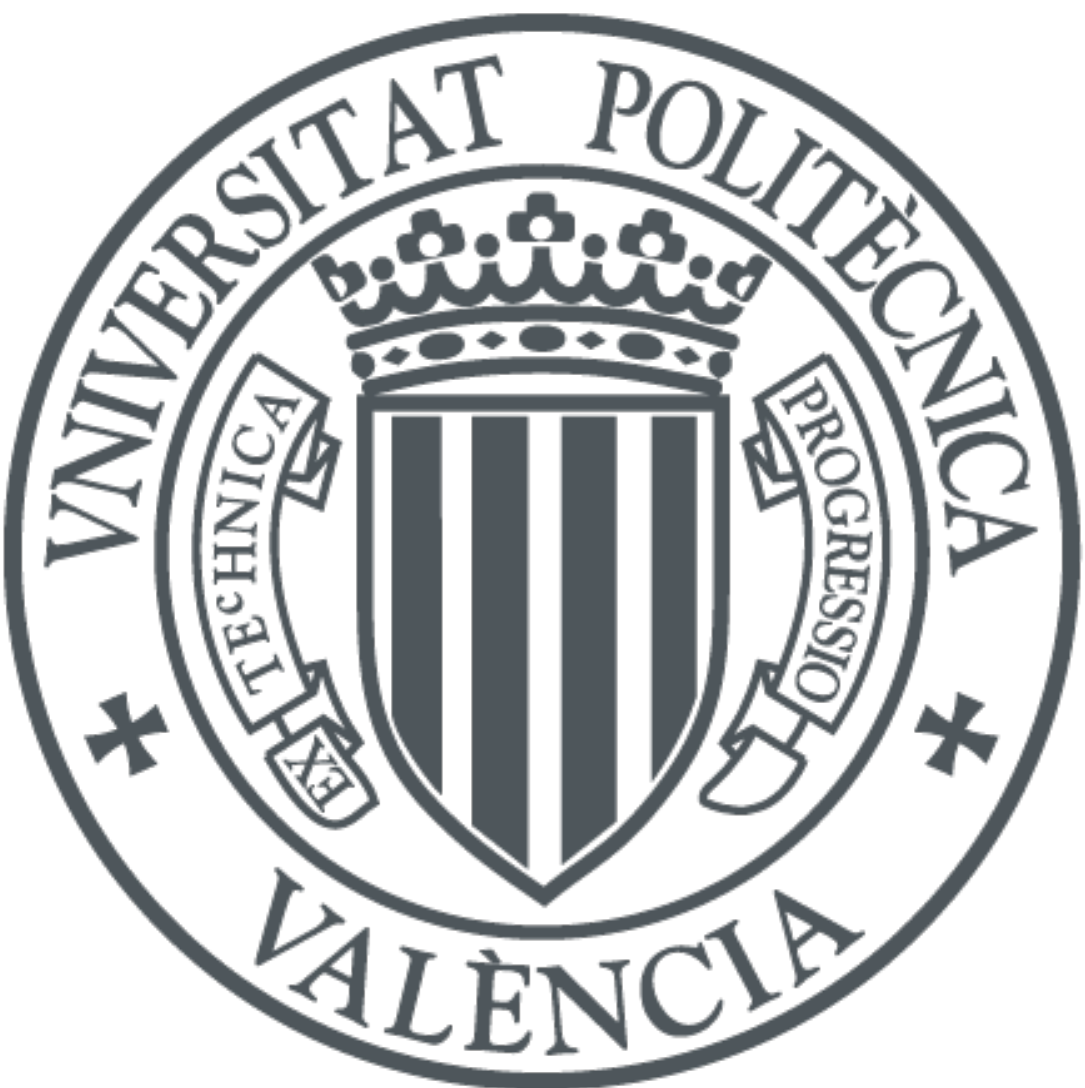

The final publication is available at

http://doi.org/10.1109/JLT.2018.2793161

Copyright Institute of Electrical and Electronics Engineers

Additional Information 


\title{
Microwave Photonics filtering interrogation technique under coherent regime for hot spot detection on a weak FBGs array
}

\author{
Javier Hervás, David Barrera, Javier Madrigal and Salvador Sales
}

\begin{abstract}
An interrogation technique of cascaded FBGs sensors based on a Microwave Photonics filtering technique under coherent regime is presented. The sensing information of a $5 \mathrm{~m}$ fiber coil containing 500 weak FBGs with a similar central wavelength is retrieved. The FBGs are $9 \mathrm{~mm}$ long and the spatial separation between consecutive FBGs is $1 \mathrm{~mm}$. The principle of operation is based on the calculation of the impulse response by recording the electrical frequency response of the system. Hot spot detection, location and temperature measurement are demonstrated by experimental measurements with a temperature sensitivity of $0.6 \mathrm{~dB} /{ }^{\circ} \mathrm{C}$ in a $10^{\circ} \mathrm{C}$ range. Fifteen measurements are recorded in order to average them to reduce noise and smooth the saw tooth appearance typical of coherent measurements. The resolution of the proposed interrogation technique is related to the bandwidth of the electrical measurement. We have obtained a resolution of $20 \mathrm{~cm}$ for an electrical bandwidth of $1 \mathrm{GHz}$. The SNR was larger than 16dB despite the fact that no amplification was used within the system.
\end{abstract}

Index Terms - Fiber Bragg gratings, optical sensors, Microwave Photonics

\section{INTRODUCTION}

$\mathrm{O}$ ptical fiber sensors have experienced an incredible growth due to the inherent low losses, light weight and electromagnetic interference immunity of the optical fiber. Additionally, the possibility to perform distributed and multiplexed sensing is another advantage compared to their electrical counterparts[1].

In quasi-distributed sensing a mass of discrete sensors are cascaded along a single fiber in order to measure environmental or structural parameters at certain given spots. Fiber Bragg Gratings (FBGs)[2] or Fabry-Perot cavities [3] are the most widely used sensing devices. Regarding FBGs, this kind of reflectors are extensively used due to both their mature fabrication technology and versatility to sense a variety of quantities, such as strain or temperature.

Manuscript received xxxxxxxx x, 2017; revised xxxxxxxx x, 2017; accepted

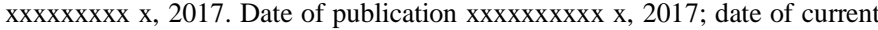
version $\operatorname{xxxxxxxx} x, 2017$. This work was supported in part by the Spanish MINECO through projects DIMENSION TEC2017-88029-R, the Generalitat Valenciana (APOSTD/2016/015), FINESSE funded by the European Union's Horizon 2020 research and innovation programme under the Marie Sklodowska-Curie Action grant agreement $\mathrm{n}^{\circ} 722509$, Sistema Nacional de Garantía Juvenil under the grant PEJ-2014-A-24709, J. Hervás work is supported by the MECD FPU scholarship (FPU13/04675)

J. Hervás, , D. Barrera, J. Madrigal and S. Sales are with the iTEAM Research Institute, Universitat Politècnica de València, Valencia 46022, Spain (e-mail: $\quad$ jaherpe2@iteam.upv.es; $\quad$ dabarvi@iteam.upv.es; jamadmad@iteam.upv.es; ssales@dcom.upv.es;).

Color versions of one or more of the figures in this paper are available online at http://ieeexplore.ieee.org

Digital Object Identifier xxxxxxxxxxxxxxxxxxxxx
Hot spots detection is also a key procedure in several application fields such as structural health monitoring or avionics [4]. The goal is to ensure the safe condition of the platform on which the sensors are located. The main techniques to retrieve the distributed sensor information are the optical time domain reflectometry (OTDR) [5] and the optical frequency domain reflectometry (OFDR) [6]. In the case of OTDR, an optical pulsed source is needed to retrieve the sensing information which is spatially resolved, whilst in the OFDR technique the wavelength of the scanning source is swept in order to locate the different sensors.

In recent years an FBG fabrication technique, known as the Draw Tower Grating (DTG) technique, has been developed. This technique is based on the inscription of the FBGs during the fiber fabrication process. The FBG sensors are continuously written using a pulsed excimer laser. The pulse repetition rate is synchronized with the fiber drawing speed. This automated production process allows to control the production parameters very accurately, thus assuring high repeatability and grating uniformity. It results in very high quality and accurately positioned FBGs. If the spatial separation between consecutive FBGs is small enough, these fibers can simulate the sensing properties of a long continuous weak FBG, also referred to as long faint FBGs [7], but with an easier fabrication process and still possessing a larger signal to noise ratio (SNR) compared to other scattering phenomena; e.g. Rayleigh [8] , Brillouin [9].

Novel interrogation techniques are being studied in order to overcome the main disadvantages of the conventional techniques $[10,11]$. The sought-after goal is to improve the resolution, sensitivity, interrogation speed, flexibility or the amount of sensors that can be interrogated in a single fiber creating this way large scale sensing networks $[12,13,14]$. The usage of microwave photonics (MWP) techniques [15] is a line of research that is attracting more and more attention from the research community. Several techniques have been presented and they proved to be able to retrieve the sensing information of point [16-19], multiplexed [10,11,20-24] and distributed photonic sensors [25].

The sensor system used in this work for hot spot detection is formed by 500 weak FBGs. The FBGs length is $9 \mathrm{~mm}$ and the grating separation is $1 \mathrm{~mm}$. The variations in the spectral properties of the FBGs are almost negligible. Traditional interrogation schemes are not suitable for the interrogation of such fibers (OTDR) [5] or have complex and costly systems (OFDR) [6]. In addition, previously presented MWP techniques used for retrieving the information of discrete reflectors show a limitation either in the number of sensors or in the spatial separation between consecutive ones. 


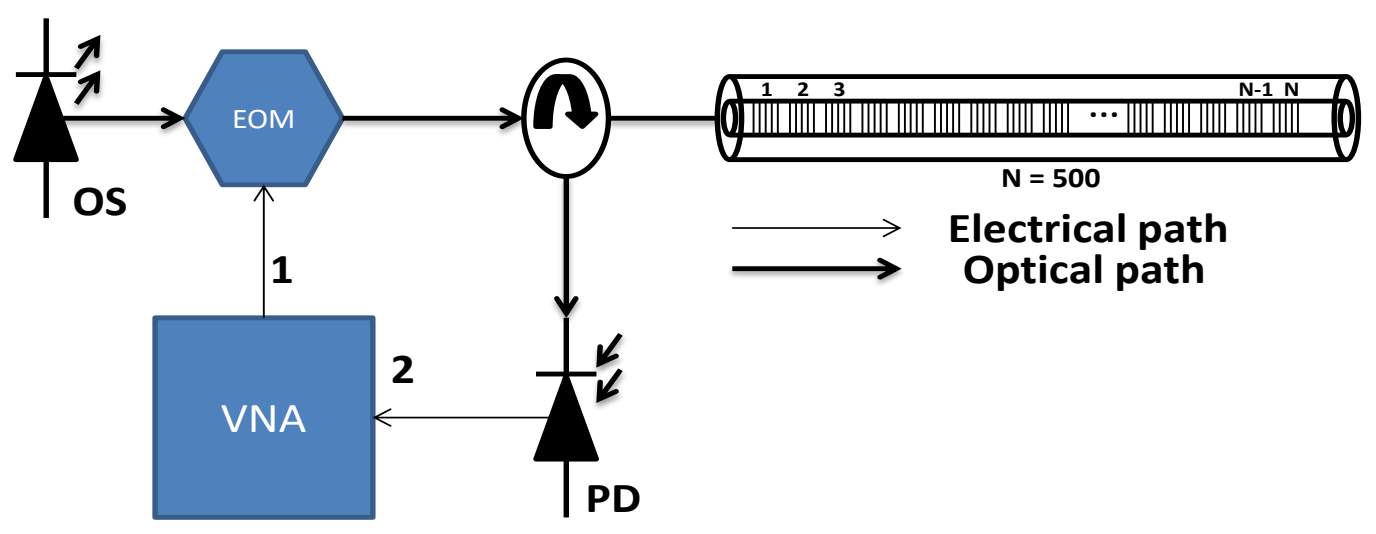

Fig.1 Schematic view of the interrogation system. OS: Optical Source; EOM: Electro-optic modulator; PD: Photodetector; VNA: Vector Network Analyzer

In this paper, we propose a microwave photonics [26] filtering interrogation technique under coherent regime [27], also known as phase-OFDR [28]. Compared with [28], there is not a requirement of using amplifiers to retrieve the backscattered light from the FBGs. Additionally, thanks to the difference in efficiency of these two scattering processes (Rayleigh scattering is an isotropic radiation process whilst FBGs couple the reflected light only in the back direction at the Bragg resonance condition), the SNR is improved and the number of averages needed for a valid measurement is almost an order of magnitude smaller. This new technique is able to retrieve the sensing information along the fiber despite the great number of sensors and the small spatial separation between consecutive gratings.

\section{PRINCIPLE OF OPERATION}

The operational principle is based on the coherent interrogation of the sensors as a MWP filter [11]. The layout of the interrogation system is shown in Fig.1. It is based on the measurement of the scattering parameter $S_{21}$. This scattering parameter is measured through the calculation of the relationship between the outgoing electrical current generated by the vector network analyzer (VNA) in port 1 with the incoming electrical current on port 2.

Initially, the light coming from an optical source (OS) is modulated in the electro-optic modulator (EOM) by the radiofrequency tones (RF-tones) generated by the VNA. This optical wave is then launched into the FBGs fiber through a circulator. The schematic design of the fiber and the superposed spectrum of all the weak FBGs can be seen in Fig.2. Finally, all the backscattered light from the FBGs is fed to the photodetector $(\mathrm{PD})$ and the generated current goes back to the VNA in order to calculate the $S_{21}$ parameter.

The electrical response of the sensor system is the summation of the samples reflected by the FBGs. The coherence time of the source and its relationship to the time delay between samples can change the appearance of the electrical spectrum. If the coherence time is smaller than the time interval between consecutive samples, there is no coherent interaction between samples, so the system works under an incoherent regime. Otherwise, the system works under a coherent regime. The incoherent regime is far more stable against environmental changes. On the contrary, a coherent regime is quite unstable but it provides more information about the sensors.

\section{EXPERIMENTAL MEASUREMENTS UNDER INCOHERENT REGIME}

The electrical response of the sensor system under incoherent regime is [11]:

$$
H(\omega)=\sum_{k=0}^{N-1} a_{k} e^{-j \Omega \tau_{k}}
$$

Where $\mathrm{N}$ is the number of reflectors, $\mathrm{a}_{\mathrm{k}}$ is the sample weight (proportional to FBG reflectivity and therefore positive), $\Omega$ is the angular frequency and $\tau_{\mathrm{k}}$ is the sample delay.

Our sensor system consists of 500 weak FBGs with almost the same spectral properties which are cascaded in a $5 \mathrm{~m}$-long fiber. These gratings have a reflectivity smaller than $0.1 \%$, so all the FBGs reflect a small amount of the incoming light. The FBGs are $9 \mathrm{~mm}$ long and the separation between consecutive gratings is $1 \mathrm{~mm}$. The time interval between samples is around 100 ps. The MWP filter formed by the 500 FBGs has a periodical electrical spectrum whose period is known as the Free Spectral Range (FSR). It is inversely proportional to the delay between consecutive samples (T), and it can be formulated as:

$$
F S R=1 / T
$$

In this case, the FSR is $10 \mathrm{GHz}$. The measured electrical spectrum is shown in Fig.3 (a). It can be observed that the spectrum shows passbands at 10 and $20 \mathrm{GHz}$. Filter theory also states that, between two consecutive FSR, the electrical response has $\mathrm{N}-1$ minima.

The FSR of the frequency response cannot change since the spatial separation between two FBGs is constant. But, as there are N-1 minima between two consecutive FSR, if one section of 

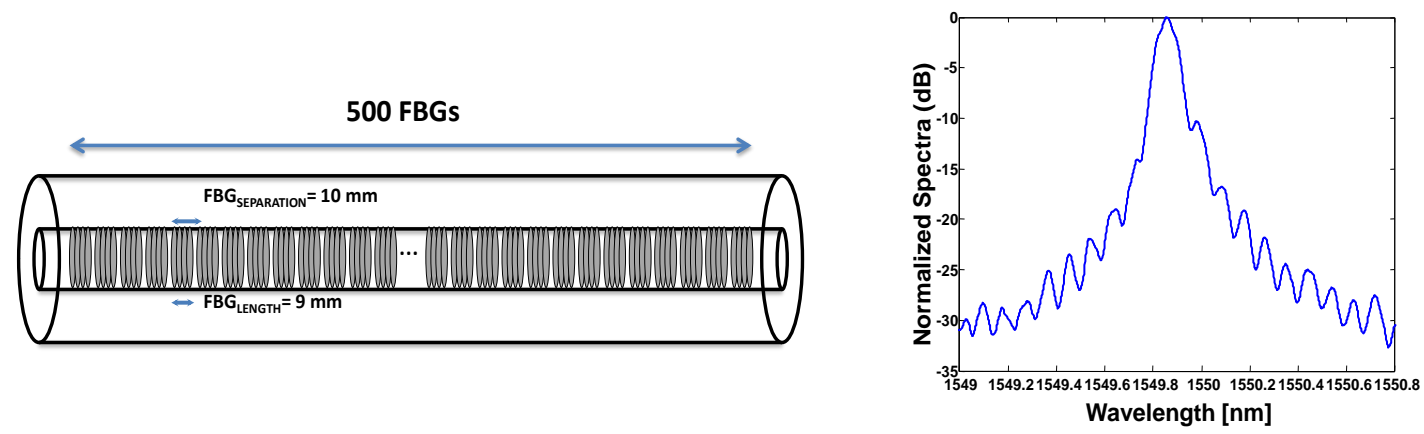

Fig. 2. Left: schematic design of the 5m fiber with 500 FBGs inscribed in it. Right: Superposed spectrum of the 500 FBGs

this fiber is heated or stretched and is selected only the reflected spectrum of the FBGs of the hot spot, the FSR would be the same but the number of samples of the filter would be smaller. In consequence, the minima spacing between two consecutive FSR is different. This was the principle of operation used in [29]. A filtered broadband source was used in order to both guarantee that the system was operating in the incoherent regime and to select only the reflected spectrum of the FBGs that were being heated/stretched and block the others. Then, the minima represent the number of FBGs ( $\mathrm{cm}$ of fiber) that are being heated/stretched. To know the position of this hot spot within the fiber, a so called reference tap had to be used. This tap was created using a physical contact (PC) connector and it was located far away from the FBGs fiber in order to be able to separate its signal from the FBGs contribution. The electrical response of the FBGs fiber plus the reference tap is shown in Fig.3(b).

Nevertheless this technique has several drawbacks. First, the position and size of the hot spot can be calculated but the temperature/strain change experienced by the FBGs cannot be extracted from the spectrum. To do so, the optical spectrum has to be studied in order to observe the Bragg wavelength change of the FBGs. This brings up a second problem. As the FBGs have the same Bragg wavelength, a large temperature/strain change has to be produced initially in order to be able to separate the contributions of the hot spot and the rest of the FBGs. This would mean that initial small changes cannot be measured by this interrogation technique. In order to overcome these disadvantages, a coherent interrogation of the same fiber is proposed.

\section{EXPERIMENTAL MEASUREMENTS UNDER COHERENT REGIME}

Working under a coherent regime means that the system is sensitive to the optical phase and as a consequence sensitive to every tiny environmental change and therefore quite unstable. To achieve a coherent regime the filtered broadband source is replaced with a laser diode (LD). This laser has a coherence time of $31.83 \mathrm{~ns}(10 \mathrm{MHz}$ linewidth). This means a coherence length of $6.36 \mathrm{~m}$, much larger than the spatial separation between consecutive FBGs. It can be observed in Fig.4 (a) that the magnitude of the electrical response seems noisy due to the coherent interference. But, although the magnitude and phase of the electrical response seem noisy, a data pattern is present since the impulse response calculated after performing an inverse fast Fourier transformation (IFFT) of the electrical response clearly shows the FBGs section of the fiber with an SNR $16 \mathrm{~dB}$ larger than the noise floor (Fig. 4 (b)). The IFFT is used to extract this data pattern easily since it cannot be extracted from the electrical spectrum (magnitude and phase) from an eye-based perspective. The impulse response is all but random (see Fig.4 (b)) and posses all the sensing information of the magnitude and phase responses. This way, the information is easily accessible. The impulse response can be formulated as:

$$
h_{B}(t)=B \sum_{k=0}^{N-1} a_{k} e^{j \pi B\left(\mathrm{t}-\tau_{k}\right)} \operatorname{sinc}\left[\mathrm{B}\left(\mathrm{t}-\tau_{k}\right)\right]
$$
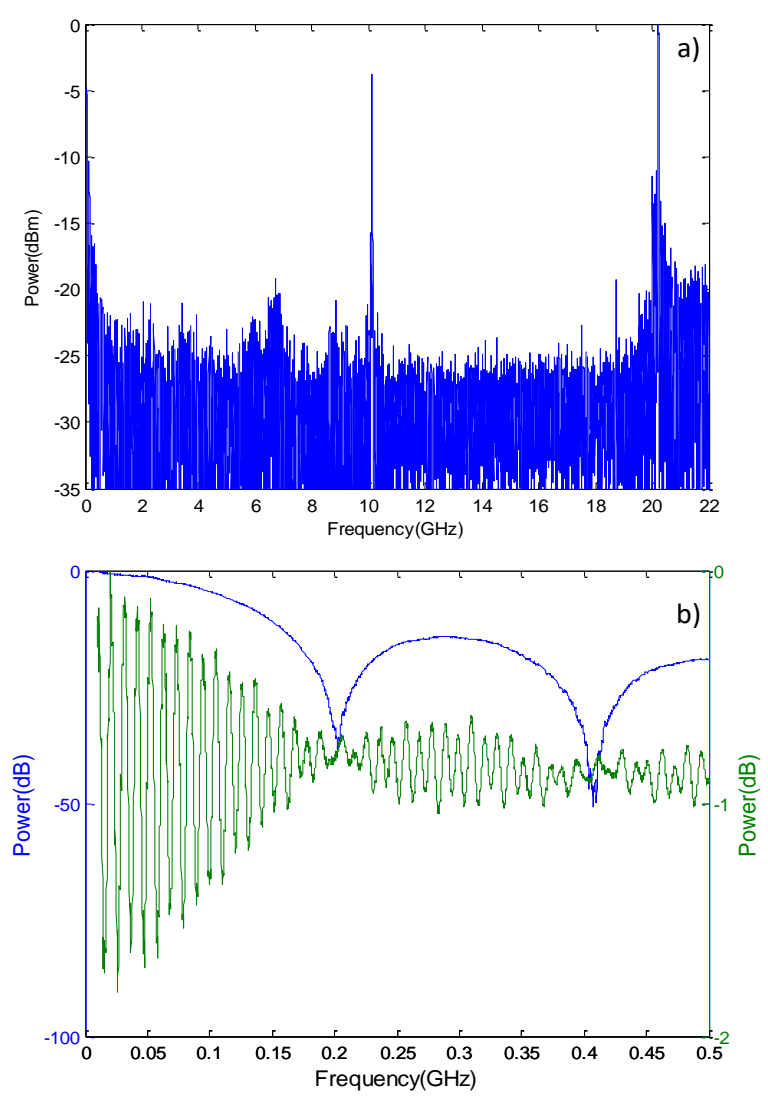

Fig.3. a) Electrical response of the $5 \mathrm{~m}$ fiber with passbands at $10 \mathrm{GHz}$ and $20 \mathrm{GHz}$. b) Zoom view of the electrical response of a hot spot (blue trace) and the same response with a reference tap (green trace) 
Where $\mathrm{B}$ is the electrical bandwidth, $\mathrm{a}_{\mathrm{k}}$ is the sample complex coefficient (related to the reflectivity of each FBG at the laser working wavelength) and $\operatorname{sinc}(\mathrm{x})=\sin (\pi \mathrm{x}) / \pi \mathrm{x}$. As can be observed in (3), every created sample by the FBGs is shown as a sinc shaped peak in the impulse response.

The impulse response shown in Fig. 4(b) has been calculated after averaging 15 impulse responses of the fiber under the same conditions in order to reduce noise and smooth the saw tooth appearance typical of coherent measurements ( 15 times is still quite small compared to Brillouin measurements as an example). As it has been stated previously, the fiber section is clearly shown with an SNR larger than $16 \mathrm{~dB}$, despite the fact that neither the optical signal nor the electrical one are amplified within the system.

The electrical response was recorded while the LD was emitting at the center Bragg wavelength of the superposed spectrum of the FBGs. Then, if a section of the fiber is heated, the Bragg wavelength of the FBGs that are located in that section will change. As a consequence the reflected power at the laser working wavelength will be smaller in these FBGs. Fig. 5 pictures it graphically. This reduction in the reflected power will be present in the impulse response. Therefore, it is possible to locate the hot spot inside the fiber coil and at the same time to measure the temperature change that the section is experiencing.
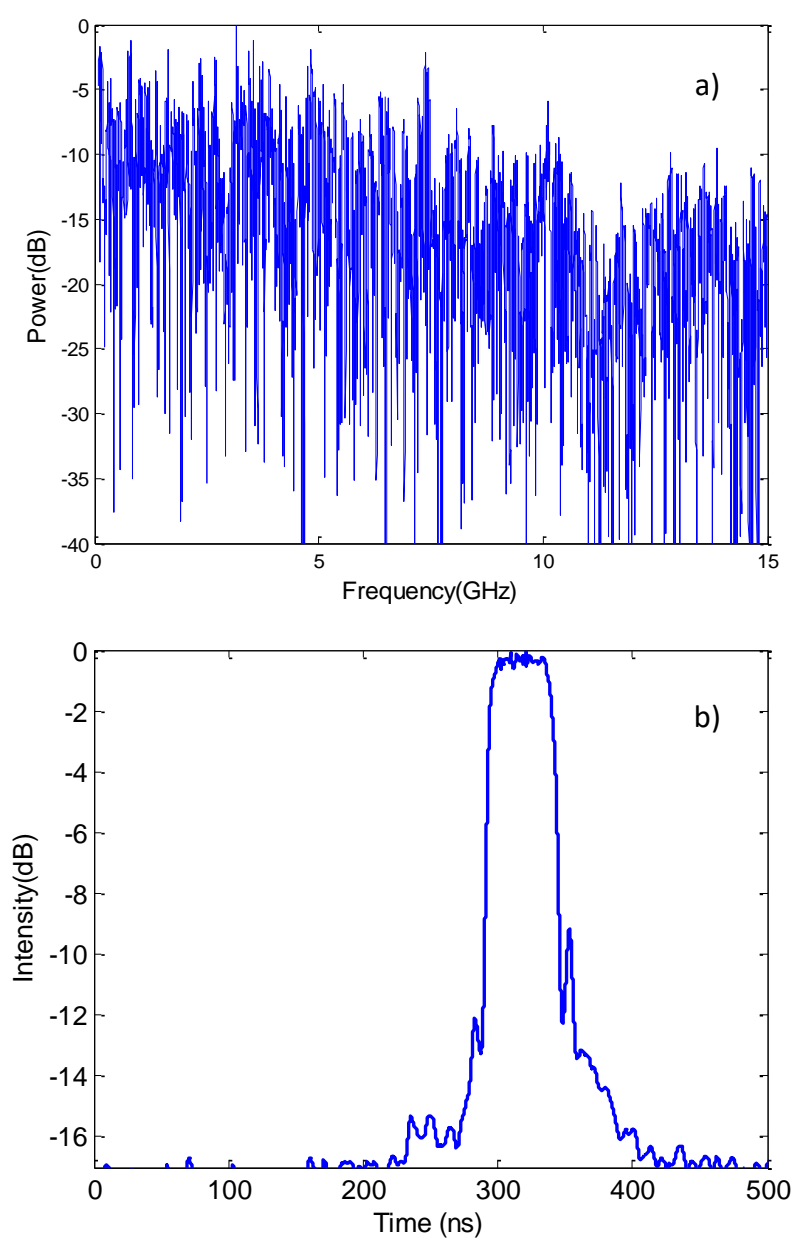

Fig. 4. a) Electrical response of the $5 \mathrm{~m}$ fiber when the system works under coherent regime. b) Impulse response of the system retrieved performing the IFFT on the magnitude and phase information of the electrical response.

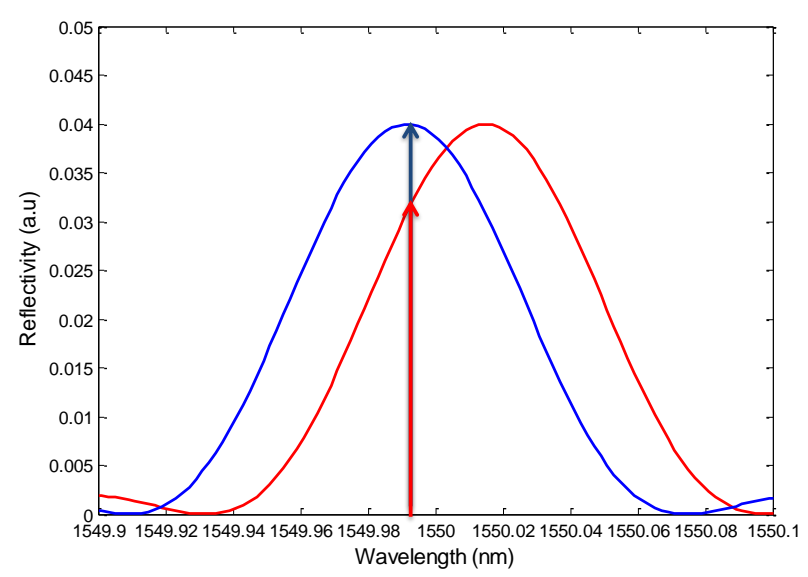

Fig. 5. Graphical representation of the principle of operation. Blue and red traces represent the spectrum of an FBG in the initial situation and when it is heated respectively. Blue and red arrows represent the reflected power at the laser working wavelength in both situations.

The setup used for the experimental measurements is depicted in Fig. 6. Two measurement sets were carried out. The first set consisted in fixing the last meters of the fiber inside a climatic chamber to perform a temperature ramp in order to calculate the sensitivity of the interrogation technique. The second set consisted in immersing a small section of the fiber in a hot bath and changing the bandwidth of the electrical spectrum measurement in order to study the relationship between this parameter and the resolution of the interrogation technique. It was established that the number of impulse responses that were going to be averaged for every temperature value was 15 . This is a tradeoff between scanning speed and quality of the measurement.

The laser was set at $1549.86 \mathrm{~nm}$ and its output power was $8 \mathrm{dBm}$. In the first measurement set, the last $2 \mathrm{~m}$ of the FBGs array were placed inside a climatic chamber with a room temperature of $22^{\circ} \mathrm{C}$. The climatic chamber has a resolution of $1^{\circ} \mathrm{C}$. Therefore, a $2^{\circ} \mathrm{C}$ step was chosen for the temperature ramp. The full temperature change range during the experiment was $10^{\circ} \mathrm{C}$. The electrical bandwidth of the VNA was set to 15 $\mathrm{GHz}$ and the spectrum was recorded with a $1 \mathrm{MHz}$ separation between consecutive points.

The results extracted from the measurements are shown in Fig.7. Fig.7 (a) shows that the intensity of the reflected signal of the last $2 \mathrm{~m}$ of fiber decreases when the temperature becomes higher. As stated earlier, this is caused by the fact that the laser is steady at the initial Bragg wavelength. So, any change in the Bragg wavelength in any FBG results in the reflectivity at the laser emitting wavelength being smaller and therefore the reflected power is smaller as well.

The averaged intensity reflected by the last two meters of fiber shows a decrement of $6 \mathrm{~dB}$ for a $10^{\circ} \mathrm{C}$ temperature change. This means that the average sensitivity of the system is $0.6 \mathrm{~dB} /{ }^{\circ} \mathrm{C}$. Additionally, Fig.7 (b), also shows that the measurement slope is not totally linear. It becomes sharper as the temperature becomes higher. This is in good agreement with the spectrum of the FBGs under study, that becomes sharper at farther wavelengths from the Bragg resonance.

In the second measurement set, $20 \mathrm{~cm}$ of the fiber located around the middle of the coil were fixed at the bottom of a small 

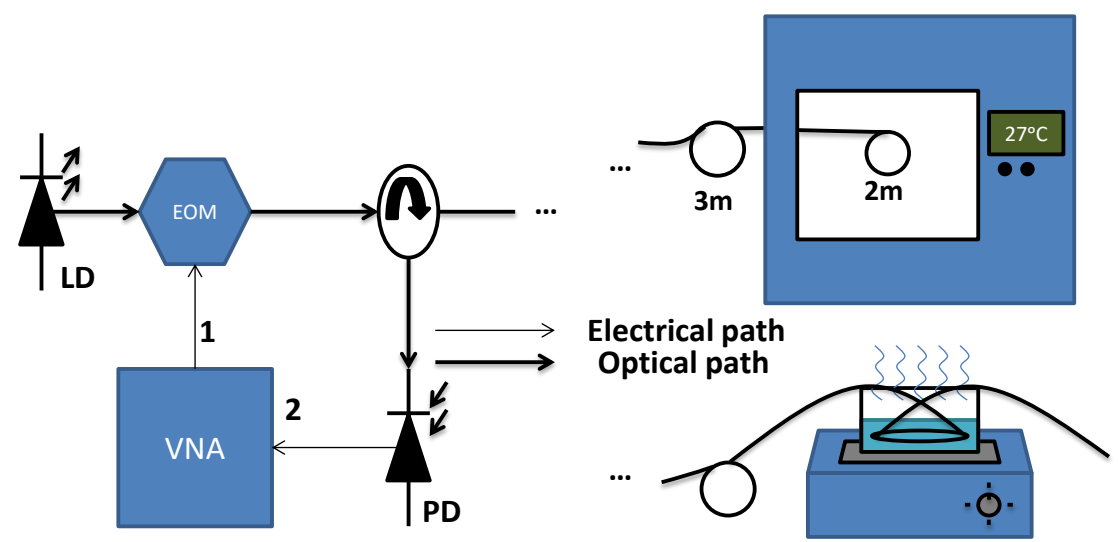

Fig. 6. Schematic view of the setup used to carry out the two set of measurements: LD: Laser Diode; EOM: Electro-Optic Modulator; PD: Photodetector; VNA: Vector Network Analyzer
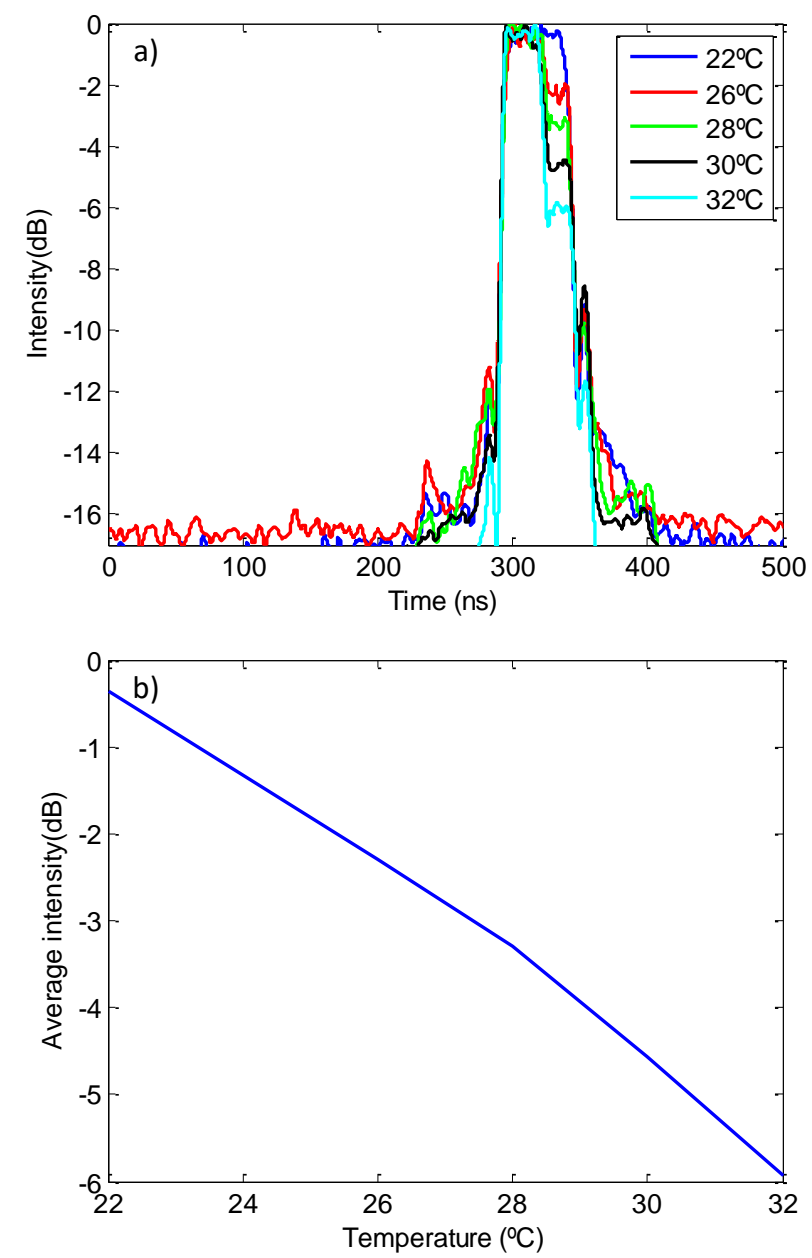

Fig. 7. Upper: Impulse response of the $5 \mathrm{~m}$ fiber for different temperatures in the hot spot. Lower: Average intensity of the $2 \mathrm{~m}$ section for different temperatures.

container in order to expose them to heated water in a hot bath. The aim was to study the effect of the electrical bandwidth in the system resolution. The electrical bandwidths of the two measurements were $15 \mathrm{GHz}$ and $1 \mathrm{GHz}$, respectively. The spectra were recorded with the same frequency step, $1 \mathrm{MHz}$.

This parameter only affects the amount of time that can be observed in the impulse response [11]. The results are shown in
Fig. 8. The spot appears as heated in both measurements but there are some differences between both of them.

The first difference is that the notch is deeper in the $1 \mathrm{GHz}$ measurement compared to the $15 \mathrm{GHz}$ one. This is due to the fact that the temperature was not being controlled and it was then different for the two distinct measurements performed. The temperature in the $15 \mathrm{GHz}$ measurement was $40^{\circ} \mathrm{C}$ whilst it was $47^{\circ} \mathrm{C}$ for the $1 \mathrm{GHz}$ one. Room temperature was $22^{\circ} \mathrm{C}$.

The second difference is that the spot seems wider for the $1 \mathrm{GHz}$ measurement. Fourier transform theory states that the distance between consecutive points in the impulse response is inversely proportional to the electrical bandwidth of the measurement. This feature limits the smallest distance between two events in order to be able to distinguish them. Furthermore, as can be extracted from (3), the width of the sinc shaped samples is determined by B. This implies that more samples are interfering in the $1 \mathrm{GHz}$ measurement at the same spot, resulting in the observation that the spot looks larger. Therefore, resolution is inversely proportional to the electrical bandwidth (higher the bandwidth the better the resolution), as in incoherent measurements [11].

This also creates the illusion that the $5 \mathrm{~m}$ fiber is slightly longer in the $1 \mathrm{GHz}$ measurement and it creates deeper ripples compared to the $15 \mathrm{GHz}$ measurement as well.

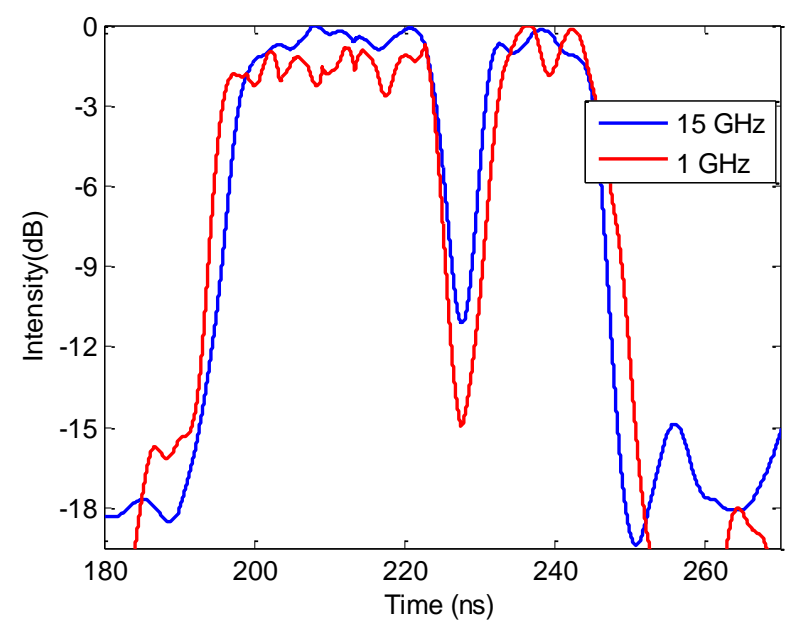

Fig. 8. Impulse response of the second measurement performed in which a $20 \mathrm{~cm}$ spot in the middle of the fiber is heated in a hot bath. 
However, the interrogation technique proves that is possible to locate hot spots inside the $5 \mathrm{~m}$ fiber and at the same time to be able to retrieve the temperature value that the fiber is experiencing in that point.

Finally, if the temperature is reduced, it would be shown in the impulse response also as an amplitude reduction. One way to ensure whether the temperature change is an increment or a decrement instead is to detune the laser wavelength just a bit $(10 \mathrm{pm})$ in order to see whether the amplitude goes up or down in this new measurement. If it goes up it means that the laser displacement follows the same trend that the Bragg wavelength change. On the contrary, if the amplitude decreases it means that laser and FBG are farther than before.

\section{CONCLUSIONS}

An interrogation technique based on a microwave photonics filtering technique under coherent regime is presented. This new technique is suitable for hot spot detection, location and temperature measurement in fibers where FBGs are written in cascade. The fiber interrogated using this technique is a 5m-long fiber including 500 FBGs. The FBGs are 9mm long and the separation between two consecutive FBGs is $1 \mathrm{~mm}$. The variations in the spectral properties of the FBGs are almost negligible. The reflectivity of the FBGs is smaller than $0.1 \%$. Therefore, each one of the FBGs reflects a small portion of the incoming light.

The principle of operation was demonstrated by means of experimental measurements whereby hot spot location and temperature measurement are proved. The resolution in this interrogation technique is inversely proportional to the bandwidth of the electrical measurement (as in incoherent measurements).

Fifteen measurements are recorded for every objective temperature. The 15 calculated impulse responses for each objective temperature were averaged in order to reduce noise and smooth the saw tooth appearance typical of coherent measurements. Despite the fact that no amplification was employed, the reflected signal from the weak FBGs was clearly shown on the averaged impulse response with an SNR larger than $16 \mathrm{~dB}$. An averaged sensitivity of $0.6 \mathrm{~dB} /{ }^{\circ} \mathrm{C}$ was demonstrated in the experimental measurements. A $20 \mathrm{~cm}$ hot spot has also been detected using an electrical bandwidth of 1 $\mathrm{GHz}$.

To increase the temperature range and the SNR, ultrashort FBGs with broader spectra and amplification can be used. Finally it is worth noting that with specific measurement devices like a tunable radio-frequency oscillator and a digital signal processor for data acquisition and analysis (performing the IFFT which nowadays is a time-effective algorithm) the sweeping speed and the results showed in this work could be improved.

\section{REFERENCES}

[1] K.T.V. Grattan, T. Sun, "Fiber optic sensor technology: an overview," Sensors and Actuators A: Physical, vol. 82, no. 1, pp. 40-61, May 2000,
[2] A. D. Kersey et al., "Fiber grating sensors," in J. Lightw. Technol., vol. 15, no. 8, pp. 1442-1463, Aug 1997.

[3] T. Yoshino, K. Kurosawa, K. Itoh and T. Ose, "Fiber-optic Fabry-Perot interferometer and its sensor applications," in IEEE J. Quantum Electron, vol. 18, no. 10, pp. 1624-1633, Oct 1982.

[4] H. Guo, G. Xiao, N. Mrad, J. Yao, "Fiber Optic Sensors for Structural Health Monitoring of Air Platforms," Sensors, vol. 11, no. 4, pp. 3687-3705, March 2011.

[5] J. Pastor-Graells, H. Martins, A. Garcia-Ruiz, S. Martin-Lopez, and M. Gonzalez-Herraez, "Single-shot distributed temperature and strain tracking using direct detection phase-sensitive OTDR with chirped pulses," Opt. Express, vol. 24, no. 12, pp. 13121-13133, May 2016.

[6] http://lunainc.com/

[7] L. Thévenaz, S. Chin, J. Sancho and S. Sales, "Novel technique for distributed fibre sensing based on faint long gratings (FLOGs)," in Proc. 23rd OFS, Santander, Spain, June 2-6, 2014, p. 91576W.

[8] H. F. Martins, S. Martin-Lopez, P. Corredera, J. D. Ania-Castañon, O. Frazão and M. Gonzalez-Herraez, "Distributed Vibration Sensing Over $125 \mathrm{~km}$ With Enhanced SNR Using Phi-OTDR Over a URFL Cavity," in J. Lightw. Technol., vol. 33, no. 12, pp. 2628-2632, June 2015.

[9] A. Dominguez-Lopez, M. Soto, S. Martin-Lopez, L. Thevenaz, and M. Gonzalez-Herraez, "Resolving 1 million sensing points in an optimized differential time-domain Brillouin sensor," Opt. Lett., vol. 42, no. 10, pp. 1903-1906, May 2017.

[10] R. Cheng, L. Xia, Y. Ran, J. Rohollahnejad, J. Zhou and Y. Wen, "Interrogation of Ultrashort Bragg Grating Sensors Using Shifted Optical Gaussian Filters," in IEEE Phot. Technol. Lett., vol. 27, no. 17, pp. 1833-1836, Sept. 2015.

[11] J. Hervás, C. R. Fernández-Pousa, D. Barrera, D. Pastor, S. Sales and J. Capmany, "An Interrogation Technique of FBG Cascade Sensors Using Wavelength to Radio-Frequency Delay Mapping," in J. Lightw. Technol., vol. 33, no. 11, pp. 2222-2227, June 2015.

[12] C. Hu, H. Wen and W. Bai, "A Novel Interrogation System for Large Scale Sensing Network With Identical Ultra-Weak Fiber Bragg Gratings," in J. of Lightw.Technol., vol. 32, no. 7, pp. 1406-1411, April1 2014.

[13] Y. Wang, J. Gong, B. Dong, D. Y. Wang, T. J. Shillig and A. Wang, "A Large Serial Time-Division Multiplexed Fiber Bragg Grating Sensor Network," in J. of Lightw. Technol., vol. 30, no. 17, pp. 2751-2756, Sept. 2012.

[14] L. Ma, C. Ma, Y. Wang, D. Y. Wang and A. Wang, "High-Speed Distributed Sensing Based on Ultra Weak FBGs and Chromatic Dispersion," in IEEE Phot. Technol. Lett., vol. 28, no. 12, pp. 1344-1347, June 2016.

[15] J. Yao, "Microwave Photonics," in J. of Lightw. Technol., vol. 27, no. 3, pp. 314-335, Feb. 2009.

[16] J. Yao, "Microwave Photonics for High Resolution and High Speed Interrogation of Fiber Bragg Grating Sensors," in Proc. of the International Photonics and OptoElectronics Meetings, Wuhan, China, June 18-21, 2014, paper OTh2C.1.

[17] C. Wang and J. Yao, "Ultrafast and Ultrahigh-Resolution Interrogation of a Fiber Bragg Grating Sensor Based on Interferometric Temporal Spectroscopy," in J. of Lightw. Technol., vol. 29, no. 19, pp. 2927-2933, Oct. 2011. 
[18] X. Dong, L. Shao, H. Fu, H. Tam, and C. Lu, "Intensity-modulated fiber Bragg grating sensor system based on radio-frequency signal measurement," Opt. Lett., vol. 33, no. 5, pp. 482-484, March 2008.

[19] R. Cheng, L. Xia, J. Yan, J. Zhou, Y. Wen and J. Rohollahnejad, "Radio Frequency FBG-Based Interferometer for Remote Adaptive Strain Monitoring," in IEEE Phot. Technol. Lett., vol. 27, no. 15, pp. 1577-1580, Aug. 2015.

[20] S. Werzinger, M. Gottinger, S. Gussner, S. Bergdolt, R. Engelbrecht and B. Schmauss, "Model-based compressed sensing of fiber Bragg grating arrays in the frequency domain," in Proc. of the 25th Optical Fiber Sensors Conference (OFS), Jeju, Korea, April 24-28, 2017, pp. 1-4.

[21] S. Werzinger, S. Bergdolt, R. Engelbrecht, T. Thiel and B. Schmauss, "Quasi-Distributed Fiber Bragg Grating Sensing Using Stepped Incoherent Optical Frequency Domain Reflectometry," in J. of Lightw. Technol., vol. 34, no. 22, pp. 5270-5277, Nov. 2016.

[22] S. Liehr and K. Krebber, "Application of Quasi-Distributed and Dynamic Length and Power Change Measurement Using Optical Frequency Domain Reflectometry," in IEEE Sensors Journal, vol. 12, no. 1, pp. 237-245, Jan. 2012.

[23] J. Huang, X. Lan, M. Luo, and H. Xiao, "Spatially continuous distributed fiber optic sensing using optical carrier based microwave interferometry," Opt. Express, vol. 22, no. 15, pp. 18757-18769, July 2014.

[24] L. Hua, Y. Song, B. Cheng, W. Zhu, Q. Zhang and H. Xiao, "Distributed optical fiber sensing based on coherence-length gated microwave photonics interferometry," in Proc of the 2017 International Topical Meeting on Microwave Photonics (MWP), Beijing, China, October 23-26, 2017, pp. 1-4.

[25] M. Koeppel, R. Engelbrecht, S. Werzinger and B. Schmauss, "Fiber sensor identification based on incoherent Rayleigh backscatter measurements in the frequency domain," in Proc. of the 25th Optical Fiber Sensors Conference (OFS), Jeju, Korea, April 24-28, 2017, pp. 1-4.

[26] J. Hervás et al., "Microwave Photonics for Optical Sensors," in IEEE J. Sel. Topics Quantum Electron., vol. 23, no. 2, pp. 327-339, March-April 2017.

[27] J. Hervás, D. Barrera, J. Madrigal, S. Sales, "Microwave photonics filtering interrogation technique under coherent regime for hot spot detection on cascaded FBG fiber," in Proc. of the 25th International Conference on Optical Fiber Sensors, Jeju, Korea, April 24-28, 2017, p. 1032341.

[28] S. Liehr and K. Krebber, "Phase-OFDR for distributed disturbance measurement," in Proc. of Sixth European Workshop on Optical Fibre Sensors, Limerick, Ireland, May 31, 2016 , p. 991624.

[29] A. L. Ricchiuti, J. Hervás, D. Barrera, S. Sales and J. Capmany, "Microwave Photonics Filtering Technique for Interrogating a Very-Weak Fiber Bragg Grating Cascade Sensor," in IEEE Photon. J., vol. 6, no. 6, pp. 1-10, Dec. 2014. 